\title{
Classification and Management Algorithm for Postoperative Wound Complications Following Transforaminal Lumbar Interbody Fusion
}

\author{
Rishi Mugesh Kanna, Karukayil Ramakrishnan Renjith, Ajoy Prasad Shetty, Shanmuganathan Rajasekaran \\ Department of Spine Surgery, Ganga Medical Centre \& Hospital Pvt. Ltd., Coimbatore, India
}

\section{Study Design: Retrospective study.}

Purpose: Postoperative wound complications occurring after transforaminal lumbar interbody fusion (TLIF) are unique, as they can involve different tissue zones (subcutaneous, subfascial, osseous, peri-implant, and disc).

Overview of Literature: Management of postoperative infections occurring after TLIF remains controversial in the context of retention or removal of implants.

Methods: A total of 1,279 consecutive patients (1,520 segments) who underwent TLIF with a minimum follow-up of 1 year were analyzed. Patients with wound complications were classified anatomically into the following five types: type 1 , suprafascial necrosis; type 2, wound dehiscence; type 3, pus around screws and rods; type 4, bone marrow edema; and type 5, pus in the disc space. Details pertaining to clinicoradiological and laboratory findings and management were also recorded.

Results: Of the 62 patients (4.8\%) with wound complications, there were seven patients in type 1, 35 in type 2, 10 in type 3, four in type 4, and six in type 5. Patients in types 1 and 2 manifested delayed wound healing and were systemically well. In type 1, five patients were managed with resuturing and two were managed conservatively. In type 2, all patients had wound gaping and were managed by debridement, whereas three patients required vacuum-assisted closure. Patients in type 3 had severe back pain and fever, with demonstrable pus around the screw site. Tissue culture identified organisms in $90 \%$ of the patients. Patients in type 4 presented with increasing back pain, and magnetic resonance imaging revealed vertebral bone marrow edema. Those in type 5 had severe back pain and fever, with demonstrable pus in the disc space. Patients in types $3-5$ required debridement, implant revision/retention, and long-term antibiotics.

Conclusions: The new anatomical classification of surgical site infections could help grade the severity of infection and provide tangible treatment guidelines, resulting in better infection clearance and patient outcomes.

Keywords: Surgical site infection; Vertebral bone marrow edema; Classification

Received Aug 4, 2019; Revised Oct 1, 2019; Accepted Oct 13, 2019

Corresponding author: Rishi Mugesh Kanna

Department of Spine Surgery, Ganga Medical Centre \& Hospital Pvt. Ltd., 313, Mettupalayam Road, Coimbatore-641043, Tamil Nadu, India

Tel: +91-9846600798, Fax: +91-422-2451444, E-mail: rishiortho@gmail.com 


\section{Introduction}

Postoperative wound complications occurring after transforaminal lumbar interbody fusion (TLIF) are potentially a serious issue. The presence of a spinal implant and interbody cage renders management more intricate. Wound complications in TLIF can involve different tissue zones, including the subcutaneous, subfascial, osseous, and disc spaces. Most published studies on the management of infections after TLIF have considered postoperative spinal infections as either superficial or deep [1-3]. None of them have mentioned about the different types of woundrelated complications (suprafascial necrosis, wound dehiscence, discitis, etc.) or about their respective management. Although the clinical presentation can be overlapping in these surgical site complications, the management protocols must be different. Because not all wound complications of TLIF are infested with microorganisms, a clear classification and a tailored management plan are essential. Moreover, even with infection, the presence and extent of infection around the implants, the vertebral body, or the disc space can necessitate widely variable management pathways. There is also a lack of consensus regarding the removal or retention or exchange of screws and cages. In this study, we reviewed the incidence and management of postoperative infections and wound complications occurring after a standard TLIF surgery and devised a pragmatic classification for the postoperative wound-related complications, which will help optimize the decisionmaking process for the practicing spine surgeon.

\section{Materials and Methods}

After obtaining written informed consent from the institutional review board of Ganga Medical Centre (IRB approval no., 26022018/GMCH/IRB), a total of 1,279 consecutive adult patients (age, 18-50 years) who underwent posterior instrumented open TLIF surgery (L2-S1 levels) for degenerative or dysplastic conditions at Ganga Medical Centre and Hospital, Coimbatore between January 2014 and June 2017 with a minimum follow-up period of 1 year were retrospectively analyzed. The treated pathologies included degenerative spondylolisthesis/instability $(n=821)$, lytic spondylolisthesis $(n=292)$, recurrent disc $(n=74)$, dysplastic listhesis $(\mathrm{n}=56)$, and revision fusion surgeries $(n=36)$. Patients who underwent fusion for nondegenerative conditions such as trauma, tumor, and infection and fusion without instrumentation or interbody cage were excluded. All patients were operated by one of two senior spine surgeons (with a post-fellowship experience of $>9$ years). A standard technique of posterior midline exposure, freehand pedicle screws, midline decompression, and interbody fusion with a single long bullet-shaped cage was used in all patients. The electronic medical record of each patient was reviewed for collecting information such as patient demographic data, medical comorbidities, primary diagnosis, number of segments fused, laboratory parameters, and wound-related complications and their management details. Patients who presented with features suggestive of an infection (subfascial wound dehiscence/ severe back pain/fever) were further evaluated by magnetic resonance imaging (MRI). Based on the anatomical zone involved, the wound complications were classified into the following five groups: type 1 , suprafascial necrosis (SN); type 2, wound dehiscence (WD); type 3, pus around screws and rods (PS); type 4, demonstrable bone marrow edema (BME); and type 5, pus in the disc space (PD).

\section{Results}

Of the 1,279 patients (women, 879; men, 400), 1,169 underwent primary surgeries and 110 underwent revision surgeries. A total of 1,068 patients had single-level fusion and 211 had multi-level fusion (two-level fusion, 181; three-level fusion, 30), with a total of 1,520 fused segments. There were 479 male and 800 female patients with a mean age of $43.2 \pm 5.6$ years. The mean duration of surgery was $141.45 \pm 42.4$ minutes, with a mean blood loss of $342.7 \pm 68.4 \mathrm{~mL}$.

In total, 62 patients $(4.8 \%)$ presented with postoperative wound-related complications, of whom there were 40 female patients $(67.7 \%)$ and 22 male patients (33.3\%). Of the 62 patients, $54(87 \%)$ presented in the early postoperative period (within 4 weeks). The incidence rate of wound complications in patients with a single-level fusion was $4.5 \%$ (48/1,068 patients) and that in patients with multi-level fusion was $6.6 \%$ (14/211 patients). The mean albumin level was $4.05 \pm 0.08 \mathrm{~g} / \mathrm{L}$. The mean erythrocyte sedimentation rate (ESR) and C-reactive protein (CRP) levels were $77.23 \pm 33.60 \mathrm{~mm} / \mathrm{hr}$ and $51.22 \pm 21.2 \mathrm{mg} / \mathrm{dL}$, respectively. Only four patients (6.4\%) underwent revision procedures. In the study group, L4-5 $(\mathrm{n}=866)$ and L5-S1 $(n=473)$ were the most commonly operated segments, followed by L3-4 and L2-3. Of the 62 patients with wound 
Table 1. The comparison of duration, blood loss, albumin levels, ESR, and CRP between the five groups

\begin{tabular}{|c|c|c|c|c|c|}
\hline Variable & Type 1 & Type 2 & Type 3 & Type 4 & Type 5 \\
\hline Duration of surgery (min) & $137.2 \pm 6.1$ & $141 \pm 4.8$ & $143.9 \pm 4.4$ & $143 \pm 4.8$ & $145.6 \pm 4.6$ \\
\hline Blood loss (mL) & $362 \pm 53.5$ & $338 \pm 72.5$ & $348 \pm 81.5$ & $345 \pm 50.6$ & $326.6 \pm 42.8$ \\
\hline Albumin (g/L) & 4.1 & 4 & 4 & 4.1 & 3.9 \\
\hline $\mathrm{ESR}(\mathrm{mm} / \mathrm{hr})$ & 65 & 106.9 & 116.5 & 41.8 & 76.2 \\
\hline $\mathrm{CRP}(\mathrm{mg} / \mathrm{dL})$ & 6.5 & 42.1 & 142.6 & 20.5 & 51 \\
\hline
\end{tabular}

Values are presented as mean \pm standard deviation or number. ESR, erythrocyte sedimentation rate; CRP, C-reactive protein.

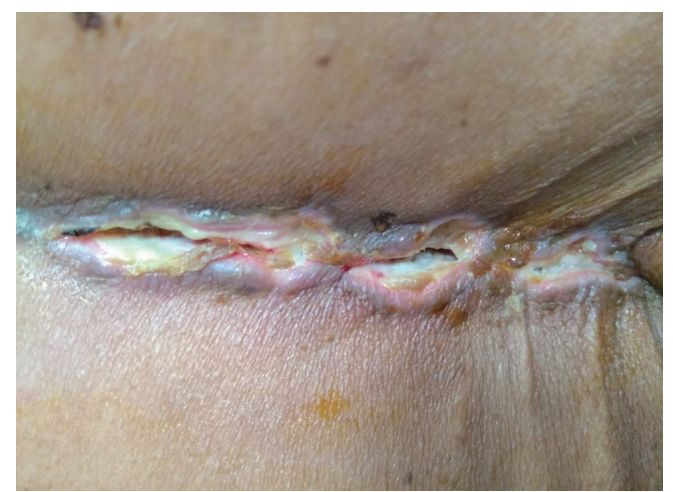

Fig. 1. The patient presented 16 days postoperatively after $L 4-5$ transforaminal lumbar interbody fusion with marginal necrosis, which was managed conservatively, and adequate wound healing was achieved in 2 weeks.

complications, L4-5 $(n=34)$ and L5-S1 $(n=25)$ were correspondingly the most commonly affected segments. The results of comparison of the duration of surgery, blood loss, albumin levels, and ESR and CRP levels between the groups are summarized in Table 1 . None of these variables were found to have a statistically significant association with postoperative wound complications, except elevated ESR and CRP levels in type 3 patients.

\section{Type 1: suprafascial necrosis}

Definition: This type included any patient with necrosis of skin and subcutaneous tissue that warranted an unplanned return to hospital and/or required a suturing (Fig. 1). Of the 62 patients, $7(11.3 \%)$ had type $1 \mathrm{SN}$ without any systemic features of infection (axial pain, fever, or purulent discharge). The mean duration from index surgery to the day of presentation was 15.4 \pm 3.2 days (range, 10-22 days). Five patients were managed with skin margin debridement and secondary suturing under local anesthesia; two patients with minimal gaping of the wound were managed conservatively with cleaning and dressing, and satisfactory wound healing was achieved in 2 weeks. Tissue culture results were negative, and none of them required antibiotic therapy or further operative treatment.

\section{Type 2: wound dehiscence}

Definition: Any patient who presented with dehiscence of the wound beyond the deep fascia with serosanguinous discharge and who required debridement under general anesthesia was classified under this group (Fig. 2).

This was the most common type of wound complication, with 35 patients $(56.4 \%)$ presenting at a mean duration of 26.2 days (range, 4-54 days) postoperatively. Although 24 patients had no other systemic signs and symptoms of infection, three patients had associated axial back pain with fever and eight had back pain alone at the time of presentation. These 11 patients underwent MRI to assess for the presence of deep foci of collection, which revealed four patients with subfascial seroma/hematoma collection and the remaining with normal postoperative changes. All patients were managed by return to the operating room, and thorough debridement was performed. Three patients required vacuum-assisted closure (VAC), followed by a successful closure of the wound. Patients with systemic signs of infection and those who underwent VAC were treated with empirical antibiotics until their serum ESR and CRP levels normalized. Tissue culture revealed organisms in four patients (Klebsiella pneumoniae, 2; coagulase-negative Staphylococcus aureus, 2) who were treated with appropriate antibiotics.

\section{Type 3: pus around implant}

Definition: This group consisted of any patient who manifested an exacerbation of postoperative surgical site pain after surgery with an MRI finding of peri-implant collec- 

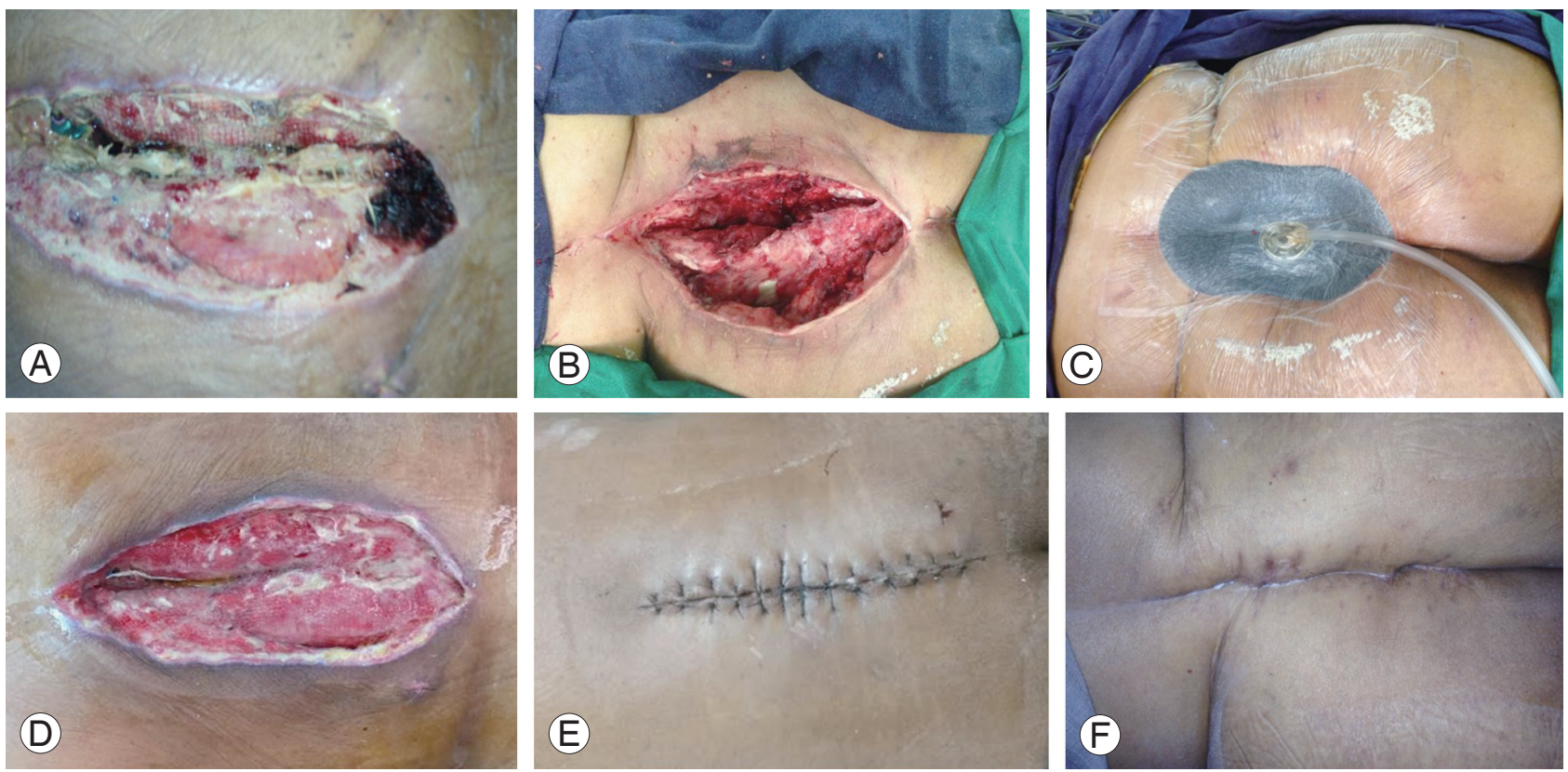

Fig. 2. The patient presented 30 days after L4-5 and L5-S1 transforaminal lumbar interbody fusion with wound dehiscence and associated serosanguinous discharge (A), managed successfully by debridement (B), vacuum-assisted closure application (C, D), and secondary suturing (E). The wound healed well after secondary suturing (F).
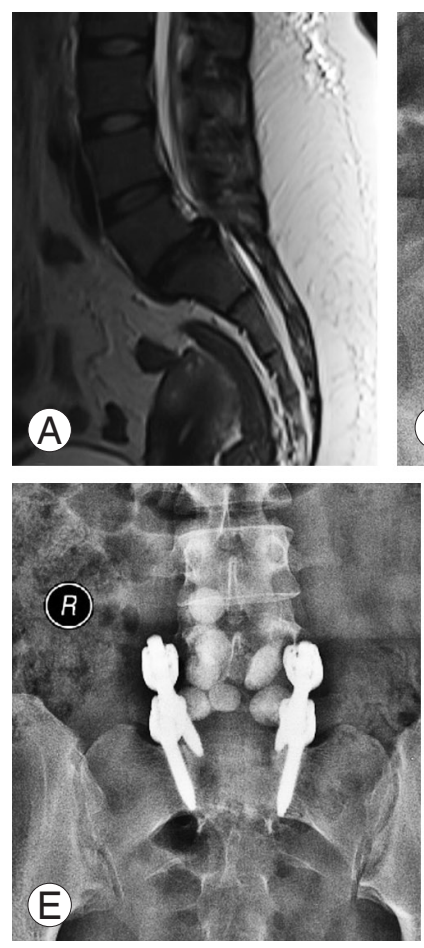

(B)
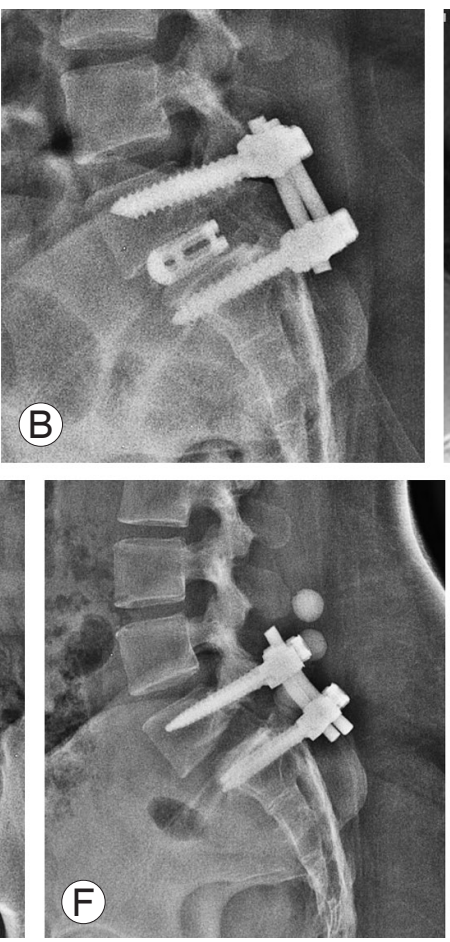

G

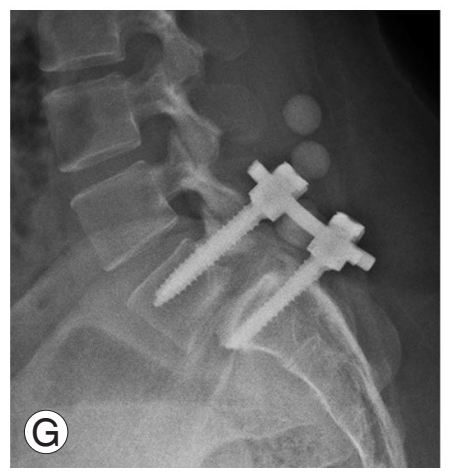

Fig. 3. This 35-year-old female patient with L5-S1 dysplastic listhesis (A) was treated with L5-S1 transforaminal lumbar interbody fusion (B). One month later, she presented with severe back pain and fever. Axial and sagittal magnetic resonance imaging (C, D) showed peri-implant collection. She was managed by debridement, screw revision, cage removal, and antibiotic-impregnated cement bead installation (E-G). At 3 months, the wound healed well and her symptoms improved.

tion confirmed by intraoperative evidence of pus (Fig. 3).

In all these patients $(n=10)$, the surgical wound had completely healed without any signs of infection or inflammation. Ten patients (16\%) presented with severe back pain and fever at a mean duration of 28.3 days (range,
8-74 days) postoperatively. All were found to have pus collected around the screw site (MRI and intraoperative findings). The mean ESR and CRP levels were grossly elevated (mean ESR, $116.5 \pm 36.4 \mathrm{~mm} / \mathrm{hr}$; mean CRP, $142.5 \pm 43.4 \mathrm{mg} / \mathrm{dL})$. Nine patients were managed with 

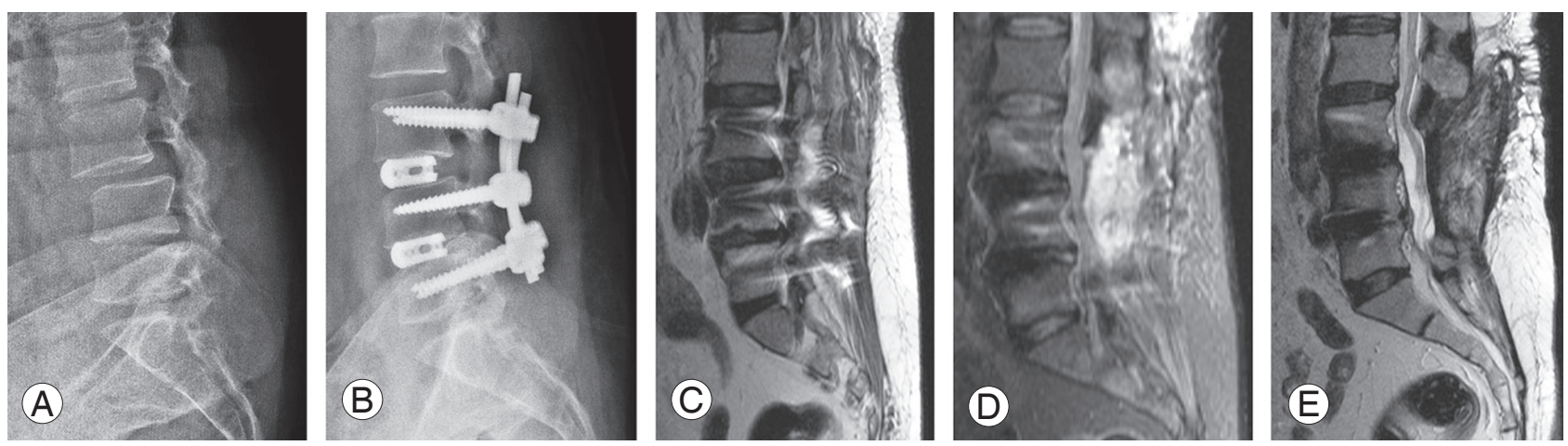

Fig. 4. This 60 -year-old female patient with $\mathrm{L} 3-4$ and $\mathrm{L} 4-5$ stenosis and instability (A) was treated with double-level transforaminal lumbar interbody fusion (B). She presented at 45 days with worsening back pain without any systemic symptoms of infection. MRI T2 and short T1 inversion recovery sagittal images showed bone marrow edema at L3 and L4 vertebral bodies (C, D). She was treated with screw revision, debridement, and antibiotics for 6 weeks. (E) Follow-up MRI at 4 months showed good healing of the inflammation. MRI, magnetic resonance imaging.

thorough debridement and implant retention. One patient required screw revision with cage removal. In addition to debridement, antibiotic-impregnated cement beads were used in two patients.

Successful closure of the wound and healing of infection with a single surgical debridement were achieved in seven patients. The two patients treated with cement beads required two further debridement procedures and another patient required VAC and three debridement procedures before achieving complete wound closure. Tissue culture and sensitivity identified organisms in 9/10 patients, and appropriate antibiotics were administered for a mean period of $6.2 \pm 2.3$ weeks. K. pneumoniae was the most common (5/10) organism isolated. Two patients each had coagulase-negative S. aureus and Escherichia coli.

\section{Type 4: bone marrow edema}

Definition: This group comprised patients who presented with delayed severe postoperative axial pain, and their MRI findings demonstrated BME in the absence of discitis and peri-implant collection (Fig. 4).

All patients $(n=4,6 \%)$ presented with an initial symptom-free interval, followed by new onset of pain. These patients manifested progressively increasing back pain at a mean duration of 35.6 days (range, 28-65 days) after surgery. Their mean ESR and CRP levels were $41.8 \mathrm{~mm} /$ $\mathrm{hr}$ and $20.2 \mathrm{mg} / \mathrm{dL}$, respectively. MRI scans demonstrated BME alone, without any fluid collection. As these patients were recalcitrant to conservative management, exploration was performed. Intraoperatively, two patients underwent revision of screws and another two patients required cage removal and revision of instrumentation (pan implant revision) because the cage was loose. Interestingly, tissue culture results showed no microbial growth in all patients. Empirical antibiotics were administered until their hematological parameters normalized. The mean duration of antibiotic therapy was $8.75 \pm 0.95$ weeks.

\section{Type 5: pus in disc space}

Definition: Any patient who presented with axial pain after surgery with the MRI demonstrating PD space with or without peri-implant collection was included in this group (Fig. 5).

Six patients (10\%) had demonstrable PD space, as evident in the postoperative MRI. All patients had persistent back pain associated with fever at a mean duration of 46.33 days (range, 33-58 days). Patients were treated with debridement and cage removal, thorough debridement of the disc space, and compression of spinal segments to aid spinal fusion. Antibiotic-impregnated cement beads were placed in the disc space in one patient, and cage revision surgery was performed in another patient 1 month later, who had persistent instability type of back pain. Tissue culture and sensitivity was reported negative in all six patients.

\section{Discussion}

TLIF procedure has become the preferred method for interbody fusion in the lumbar spine [4]. However, varying incidences of complications such as infection, accidental durotomy, screw malposition, cage migration, retroperi- 



Fig. 5. This 62-year-old female patient presented with double-level lytic spondylolisthesis at L4-5 and L5-S1 (A). She underwent two-level transforaminal lumbar interbody fusion (B). After 40 days, she reported severe axial pain in the lumbar spine, and her magnetic resonance imaging showed pus collection in the disc space extending into the lateral recess (arrow) (C) and the epidural space (D). She underwent debridement, cage revision, and antibiotic therapy. Follow-up radiographs at 8 months showed good healing and fusion (arrow) (E).

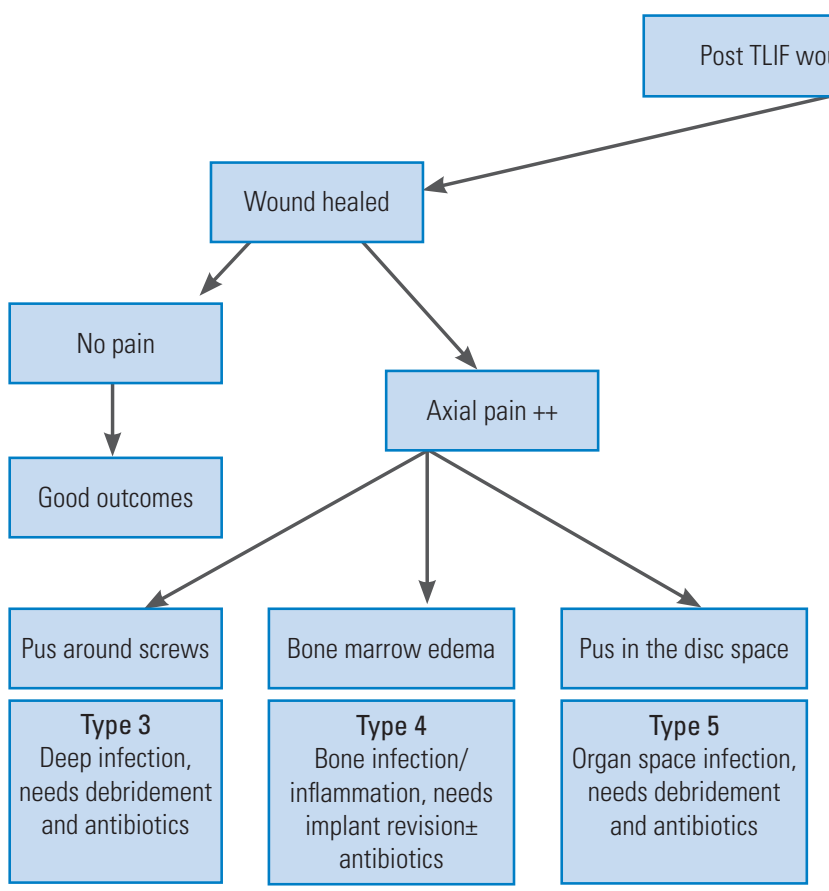

The algorithm for clinical presentation, evaluation, and management of various types of wound complications and infections occurring after TLIF surgeries. TLIF, transforaminal lumbar interbody fusion.

toneal injury, wrong level surgery, and neurological deficit have also been reported [5]. Development of infection after a TLIF procedure is a serious, cost-consuming, and rarely life-threatening complication. In the past 10 years, 
Table 2. Summary of management of all five groups

\begin{tabular}{|c|c|c|c|c|}
\hline Type & No. $(\%)$ & Interventions required & Implant removal/revision & Tissue culture and sensitivity \\
\hline 1. Supra-fascial necrosis & $7(11.3)$ & Single: 5 & 0 & 0 \\
\hline 2. Wound dehiscence & $35(56.4)$ & $\begin{array}{l}\text { Single: } 34 \text {, multiple: } 3 \text { (vacuum- } \\
\text { assisted closure) }\end{array}$ & 0 & $\begin{array}{l}\text { 4/35; Staphyloccus aureus: 2, Klebsiella } \\
\text { pneumoniae: } 2\end{array}$ \\
\hline 3. Pus around screws & $10(16)$ & Single: 7, multiple: 3 & Screw revision: 1 & $\begin{array}{l}\text { 9/10; Staphyloccus aureus: 2, Klebsiella } \\
\text { pneumoniae: } 5, \text { Escherichia coli: } 2\end{array}$ \\
\hline 4. Bone marrow edema & $4(6)$ & Single: 4 & $\begin{array}{l}3(75 \%) \text {; screw revision: } 2, \text { cage } \\
\text { removal+screw revision: } 2\end{array}$ & 0 \\
\hline 5. Pus in disc space & $6(10)$ & Single: 4, multiple: 2 & $\begin{array}{l}6(100 \%) \text {; cage revision: } 1 \text {, cage } \\
\text { removal: } 5\end{array}$ & 0 \\
\hline
\end{tabular}

the reported rates of surgical site infections from individual surgeons or institutions following elective thoracic or lumbar spinal arthrodesis have ranged from $1.9 \%$ to $4.4 \%$ [6-10]. Our study found a wound complication rate of $4.8 \%$, which closely corresponds with the previously reported rate, although subtypes 1 and 4 are likely to be noninfective.

Our study showed that wound complications occurring after TLIF do not belong to a single type (Fig. 6, Table 2). Patients in types 1 and 2 presented with primary wound problems and did not have frank systemic findings of infection. Based on whether it is suprafascial (type 1) or subfascial (type 2) dehiscence, an appropriate decision was taken to manage the wound. Suprafascial dehiscence is either conserved or resutured under local anesthesia. However, subfascial wounds require thorough debridement and closure. Short-term VAC therapy may also be required in patients with recurrent dehiscence or an obese body habitus. Typically, these patients healed well and did not require either implant removal or any antibiotic therapy.

Patients in types 3, 4, and 5 were more ominous in their symptom presentation. These patients had a delay in presentation from the time of surgical intervention. Typically, increasing back pain disproportionate to the surgical site pain was the classical presenting symptom. Patients in types 3 and 5 had pus collection around the implants. This was evident in the MRI scan and confirmed intraoperatively. Patients in type 5 showed pus collection in the disc space, making them the most severely infected among this group. Patients in type 4 had severe BME within the vertebrae adjacent to the fused disc. Although there was no pus collection, these patients presented with severe axial pain. Thus, we suggest that MRI scan is essential to identify the type of infection in a patient who presents with severe ax- ial pain after an initial pain-free period. Although clinical presentation is similar in all the three subtypes, the management is variable. Patients in type 3 required thorough debridement and implant revision while the cage could be retained, whereas those in type 5 required cage removal and thorough debridement of the disc space. It is worthwhile to note that blood parameters were also not helpful in diagnosing types $3-5$, although patients in type 3 had grossly elevated ESR and CRP levels.

Most postoperative wound infections occurring after posterior instrumented fusions can be eradicated by prompt recognition, aggressive debridement and irrigation, primary or delayed closure, and appropriate antibiotic therapy. However, controversy remains regarding implant removal versus retention, especially in early-onset infections. In a review of 28 infected spinal fusions, Wimmer and Gluch [11] in 1996 stated that the acute phase of infection could be successfully treated without removing the implants, whereas the chronic phase of infection always required implant removal. In contrast, Picada et al. [12] in 2000 successfully treated 24 of 26 deep wound infections after instrumented lumbosacral fusions by preserving instrumentation. In 2007, Mirovsky et al. [13] also treated infected PLIF without removing the interbody cages. However, after a retrospective review of 26 delayed infections following spinal deformity surgery, Hedequist et al. [14] in 2009 reported that infection could not be cleared in any patient without implant removal.

A major reason for this controversy might be that various authors have reviewed infections of varying severity. The majority of published studies have considered infections as either superficial or deep without considering the different tissue zones involved [1-3]. To solve this controversy, we classified the wound-related complications into five groups based on anatomy, in the ascending order of 
severity, and devised the management strategy for each subtype. To the best of our knowledge, none of the earlier publications have described a detailed anatomical classification for postoperative spinal infections, which would help in decision-making. According to our classification, Types 1 and 2 represented superficial zones, most of these patients were managed by simple debridement, resuturing, and short-term antibiotics with retention of implants. However, patients in types 3-5 manifested a more serious presentation and were assessed predominantly on the basis of MRI findings. The reliability of MRI in diagnosing postoperative infections and optimizing decision-making has been reported in a recent publication [15]. Of the 10 patients in types 4 and 5, eight required cage removal. In type 3 , nine of the 10 patients were managed with debridement alone.

The isolation of the causative organism was also considerably evident in type 3 infections with pus around the screws. These could represent the classical postoperative deep wound infections. Patients in type 4 with BME could manifest a metallurgical response to implanted screws and cage or a low-virulent organism, because the infective organism was not isolated in any of these patients. Similarly, in type 5 infections, despite the presence of PD space, none of the cultures grew the infective organism. Because recent studies indicate the presence of multiple low-virulent organisms in the disc space diagnosed by high-end investigations such as proteomics and genomic studies, such fastidious organisms could be the source of types 4 and 5 post-TLIF infections [16,17]. We assume that the use of extended culture tests and cutting-edge investigations can be applied in such patients to identify the pathogens in such situations.

This study is not without limitations. First, it was a retrospective study with a relatively shorter follow-up period. Second, although the results were consistent with the literature review $[1,18-21]$, the results and the ensuing classification must be validated in a prospective study. Third, the number of patients was small in certain subtypes, which preclude any significant statistical analysis to be performed. A large-scale multicenter study would be helpful. Despite these limitations, this study has several key observations. It is evident that instrumentation removal is not necessary in acute infections unless the patients belong to type 4 or 5 , which can be considered as an organ-space infection. The involvement of the disc space or end-plates warrants extensive debridement with cage removal. In delayed infections, pan implant removal can be performed. In type 3, screw removal or revision is required only when there is loosening. Antibiotic-impregnated cement beads and VAC therapy are useful options in tissues with questionable viability before achieving complete wound closure [22].

\section{Conclusions}

Wound complications occurring after TLIF are versatile with varying clinical and radiological findings that correspond to the afflicted anatomical zones. Thus, the management principles are correspondingly different. Our new anatomical classification of postoperative infections could help in grading the severity of infection and provide appropriate treatment guidelines. It is generally possible to treat adequately an early infection while retaining the spinal instrumentation, provided the disc space and the vertebral end-plates remain unaffected.

\section{Conflict of Interest}

No potential conflict of interest relevant to this article was reported.

\section{Author Contributions}

Conceptualization: RMK, KRR, APS, SR; data curation and software: RMK; formal analysis: RMK, KRR, APS, SR; methodology: RMK, KRR, APS; writing-original draft: RMK, KRR, APS; writing-review \& editing: SR; supervision and validation: KRR, APS; project administration: SR; and final approval of the version: RMK, KRR, APS, SR

\section{References}

1. Weinstein MA, McCabe JP, Cammisa FP Jr. Postoperative spinal wound infection: a review of 2,391 consecutive index procedures. J Spinal Disord 2000;13:422-6.

2. Christodoulou AG, Givissis P, Symeonidis PD, Karataglis D, Pournaras J. Reduction of postoperative spinal infections based on an etiologic protocol. Clin Orthop Relat Res 2006;444:107-13.

3. Chaudhary SB, Vives MJ, Basra SK, Reiter MF. Postoperative spinal wound infections and postprocedural diskitis. J Spinal Cord Med 2007;30:441-51. 
4. Goz V, Weinreb JH, Schwab F, Lafage V, Errico TJ. Comparison of complications, costs, and length of stay of three different lumbar interbody fusion techniques: an analysis of the Nationwide Inpatient Sample database. Spine J 2014;14:2019-27.

5. Tormenti MJ, Maserati MB, Bonfield CM, et al. Perioperative surgical complications of transforaminal lumbar interbody fusion: a single-center experience. J Neurosurg Spine 2012;16:44-50.

6. Fang A, Hu SS, Endres N, Bradford DS. Risk factors for infection after spinal surgery. Spine (Phila $\mathrm{Pa}$ 1976) 2005;30:1460-5.

7. Olsen MA, Mayfield J, Lauryssen C, et al. Risk factors for surgical site infection in spinal surgery. J Neurosurg 2003;98(2 Suppl):149-55.

8. Olsen MA, Nepple JJ, Riew KD, et al. Risk factors for surgical site infection following orthopaedic spinal operations. J Bone Joint Surg Am 2008;90:62-9.

9. Glassman SD, Dimar JR, Puno RM, Johnson JR. Salvage of instrumental lumbar fusions complicated by surgical wound infection. Spine (Phila Pa 1976) 1996;21:2163-9.

10. Pull ter Gunne AF, Cohen DB. Incidence, prevalence, and analysis of risk factors for surgical site infection following adult spinal surgery. Spine (Phila Pa 1976) 2009;34:1422-8.

11. Wimmer C, Gluch H. Management of postoperative wound infection in posterior spinal fusion with instrumentation. J Spinal Disord 1996;9:505-8.

12. Picada R, Winter RB, Lonstein JE, et al. Postoperative deep wound infection in adults after posterior lumbosacral spine fusion with instrumentation: incidence and management. J Spinal Disord 2000;13:425.

13. Mirovsky Y, Floman Y, Smorgick Y, et al. Management of deep wound infection after posterior lumbar interbody fusion with cages. J Spinal Disord Tech 2007;20:127-31.
14. Hedequist D, Haugen A, Hresko T, Emans J. Failure of attempted implant retention in spinal deformity delayed surgical site infections. Spine (Phila Pa 1976) 2009;34:60-4.

15. Kanayama M, Hashimoto T, Shigenobu K, Oha F, Iwata A, Tanaka M. MRI-based decision making of implant removal in deep wound infection after instrumented lumbar fusion. Clin Spine Surg 2017;30:E99-103.

16. Rajasekaran S, Tangavel C, Aiyer SN, et al. ISSLS Prize in Clinical Science 2017: is infection the possible initiator of disc disease?: an insight from proteomic analysis. Eur Spine J 2017;26:1384-400.

17. Rajasekaran S, Tangavel C, K S SVA, et al. Inflammaging determines health and disease in lumbar discs-evidence from differing proteomic signatures of healthy, aging, and degenerating discs. Spine J 2020;20:48-59.

18. Koutsoumbelis S, Hughes AP, Girardi FP, et al. Risk factors for postoperative infection following posterior lumbar instrumented arthrodesis. J Bone Joint Surg Am 2011;93:1627-33.

19. Sierra-Hoffman M, Jinadatha C, Carpenter JL, Rahm M. Postoperative instrumented spine infections: a retrospective review. South Med J 2010;103:25-30.

20. Pappou IP, Papadopoulos EC, Sama AA, Girardi FP, Cammisa FP. Postoperative infections in interbody fusion for degenerative spinal disease. Clin Orthop Relat Res 2006;444:120-8.

21. Viola RW, King HA, Adler SM, Wilson CB. Delayed infection after elective spinal instrumentation and fusion: a retrospective analysis of eight cases. Spine (Phila Pa 1976) 1997;22:2444-50.

22. Karaaslan F, Erdem S, Mermerkaya MU. Wound management with vacuum-assisted closure in postoperative infections after surgery for spinal stenosis. Int Med Case Rep J 2014;8:7-11. 